\title{
Aplicação da escala de depressão geriátrica abreviada em idosos ativos e sedentários do HIPERDIA
}

\author{
Application of the abbreviated geriatric depression scale in active and sedentary elderly \\ people of the HIPERDIA
}
Aplicación de la escala abreviada de depresión geriátrica en personas mayores activas y sedentarias de lo HIPERDIA
Maiara Goudim de Melo ${ }^{1 *}$, Rômulo Pinheiro Santos ${ }^{1}$, Sandra dos Santos Tavares ${ }^{1}$, Adriana Paiva Camargo Saraiva ${ }^{1}$, Anny Karoliny Nascimento Carvalho ${ }^{1}$, Joaquim Henrique Ribeiro Pereira ${ }^{1}$, Fernanda Cristina da Costa Silva'.

\section{RESUMO}

Objetivo: Aplicar a escala de depressão geriátrica em idosos ativos e sedentários do programa HIPERDIA. Métodos: Trata-se de estudo transversal descritivo, quantitativo, aprovado por Comitê de Ética em Pesquisa, com aplicação de questionário sociodemográfico e Escala de Depressão Geriátrica Abreviada (EDGA) para idosos com 60 anos ou mais, portadores de hipertensão e/ou diabetes cadastrados no programa HIPERDIA de uma Estratégia Saúde da Família. Os dados foram analisados utilizando o teste do Qui-quadrado de Person e Denver II com nível de significância de $p$-valor $<0,05$ por meio do software IBM SPSS Statistic versão 24.0. Resultados: a maioria significativa dos idosos são do sexo feminino $(32 ; 54,2 \%)$, casados $(27 ; 45,8 \%)$, possuem filhos $(56 ; 94,9 \%)$, hipertensão $(31 ; 52,5 \%)$, não são ativos $(34 ; 57,6 \%)$ e apresentaram escore EDGA entre 1 e $5(41 ; 69,5 \%)$, indicando ausência de depressão. Os idosos que praticam exercício físico regularmente têm 32 vezes mais chances de ausência de depressão; os que possuem filhos ou moram com um parceiro têm 3 vezes mais chances de ausência de depressão. Conclusão: Na população estudada, praticar exercício físico, morar com um parceiro e ter filhos parece impactar positivamente o enfrentamento de situações de estresse.

Palavras-chave: Idoso, Depressão, Exercício físico.

\begin{abstract}
Objective: To apply the geriatric depression scale in active and sedentary elderly people of the HIPERDIA program. Methods: This is a cross-sectional, descriptive, quantitative study, approved by the Research Ethics Committee, with the application of a sociodemographic questionnaire and an Abbreviated Geriatric Depression Scale (AGDS) for elderly people aged 60 or over, with hypertension and / or diabetes of the HIPERDIA program of a Family Health Strategy. The data were analyzed using the Chi-square test of Person and Denver II with a significance level of $p<0.05$ using IBM SPSS Statistic software version 24.0. Results: the significant majority of the elderly are female $(32 ; 54.2 \%)$, married $(27 ; 45.8 \%)$, have children $(56 ; 94.9 \%)$, hypertension ( 31 ; $52.5 \%)$, are not active $(34 ; 57.6 \%)$ and had an AGDS score between 1 and $5(41 ; 69.5 \%)$, indicating absence of depression. Elderly people who exercise regularly are 32 times more likely to have no depression; those who have children or live with a partner are 3 times more likely to have no depression. Conclusion: In the population studied, practicing physical exercise, living with a partner and having children seems to positively impact coping with stressful situations.
\end{abstract}

Keywords: Elderly, Depression, Physical exercise.

${ }^{1}$ Universidade do Estado do Pará (UEPA), Conceição do Araguaia - PA. *E-mail: adriana.saraiva@uepa.br SUBMETIDO EM: 6/2020 


\section{RESUMEN}

Objetivo: aplicar la escala de depresión geriátrica en ancianos activos y sedentarios del programa HIPERDIA. Métodos: Estudio transversal, descriptivo, cuantitativo, aprobado por el Comité de Ética en Investigación, con la aplicación de un cuestionario sociodemográfico y una Escala Abreviada de Depresión Geriátrica (EADG) para ancianos de 60 años o más, con hipertensión arterial y / o diabetes del programa. HIPERDIA de una estrategia de salud familiar. Los datos se analizaron usando la prueba de Chi-cuadrado de Person y Denver II, con un nivel de significancia de $p<0.05$, usando el software IBM SPSS Statistic versión 24.0. Resultados: la mayoría significativa de los ancianos son mujeres (32; $54.2 \%$ ), casadas $(27 ; 45.8 \%)$, tienen hijos (56; $94.9 \%)$, hipertensión $(31 ; 52.5 \%)$, no está activo $(34 ; 57.6 \%)$ y tenía una puntuación EADG entre 1 y 5 ( 41 ; $69.5 \%$ ), lo que indica ausencia de depresión. Los ancianos que hacen ejercicio regularmente tienen 32 veces más probabilidades de no tener depresión; quienes tienen hijos o viven con una pareja tienen tres veces más probabilidades de no tener depresión. Conclusión: En la población estudiada, practicar ejercicio físico, vivir con una pareja y tener hijos parece tener un impacto positivo para hacer frente a situaciones estresantes.

Palabras clave: Ancianos, Depresión, Ejercicio físico.

\section{INTRODUÇÃO}

Nas últimas décadas, têm-se presenciado alterações demográficas significativas no Brasil e no mundo, marcadas pelo aumento da expectativa de vida do ser humano que ocorre em concomitância a uma redução nos índices de natalidade, a consequência de tal fenômeno é um processo de envelhecimento populacional progressivo (GIL IMA, et al., 2018).

Silva MR, et al. (2018) afirmam que nas próximas décadas o Brasil passará de um contexto de mortalidade típico de populações jovens, para um quadro composto por patologias típicas de países longevos, caracterizado por doenças complexas e múltiplas, que além de perdurarem por anos, requerem cuidados contínuos e constantes.

Desta forma, com ao passar dos anos haverá maior incidência de doenças de caráter crônico, pois com o processo de envelhecimento, é natural que o organismo passe por uma série de transformações que culminam em uma redução das capacidades biológicas e mudanças na fisiologia de órgãos e sistemas. Dessa forma, a população idosa fica mais vulnerável, assim, tornando-se mais predisposta a adquirir doenças crônicas (NERY BLS, et al., 2018).

Dentre as diversas morbidades de caráter crônico que mais afetam a população idosa, encontra-se a depressão, que tem demonstrado ser um grave problema de saúde pública, acometendo cada vez mais pessoas ao longo dos anos. De acordo com o estudo de Amaral TLM, et al. (2018), esse distúrbio atinge em escala global cerca de $0,9 \%$ a $9,4 \%$ dos idosos sadios e de $14 \%$ a $42 \%$ dos idosos doentes, no Brasil, a prevalência da depressão nessa população, varia de 19\% a 34\% nos mais variados estados do país.

Nessas condições, os idosos realizam o tratamento convencional para a depressão através do uso de antidepressivos, portanto, vale ressaltar que esses medicamentos podem estar associados ao surgimento de implicações recorrentes, tais como efeitos colaterais, reações adversas, desenvolvimento de dependência, polifarmácia, fragilização do idoso, e especialmente preocupante, interações medicamentosas, como no caso de idosos portadores de condições crônicas, como hipertensão e diabetes (AGUIAR BM, et al., 2019; NASCIMENTO PPP e BATISTONI SST, 2019).

Neste contexto, surge a necessidade de adoção de medidas complementares à farmacoterapia tradicional usada no tratamento da depressão e que busquem melhorar a qualidade de vida desses idosos. Silva VRR, et al. (2015), demonstram os benefícios que o exercício físico é capaz de proporcionar aos idosos que o praticam regularmente, pois agem na diminuição dos estressores que causam depressão, ansiedade e transtornos do humor, permitindo autonomia e a realização das atividades de vida diária. 
Pesquisas apontam que o exercício físico é uma alternativa não farmacológica eficaz na prevenção e tratamento da depressão, pois tal prática, quando realizada de forma regular, está associada ao aumento da liberação catecolaminas como a serotonina, noradrenalina, dopamina e de endorfinas, que propiciarão a sensação de bem-estar, promovendo dessa forma, a sensação de disposição, contentamento e o alivio dos sinais e sintomas depressivos, nessa perspectiva, de acordo com a literatura pesquisada, aqueles idosos que possuem um estilo de vida ativo, tendem a apresentar menores chances de desenvolver transtorno depressivo, quando comparado com idosos considerado sedentários (SILVA MR, et al., 2018; ALMEIDA E, et al., 2018; CAMBOIM FEF, et al., 2017; RODRIGUES RAP, et al., 2018).

Sob a hipótese de que idosos ativos apresentarão mais chances de ter ausência de depressão, o presente estudo objetiva aplicar a Escala de Depressão Geriátrica Abreviada (EDGA) entre idosos ativos e sedentários do HIPERDIA para verificar o impacto do exercício físico no resultado da EDGA e aspectos sociodemográficos em idosos de uma Estratégia Saúde da Família (ESF) do município de Conceição do Araguaia-PA.

\section{MÉTODOS}

Trata-se de um estudo transversal descritivo, com abordagem quantitativa, onde realizou-se aplicação de questionário sociodemográfico e da Escala de Depressão Geriátrica Abreviada (EDGA) entre idosos ativos e sedentários cadastrados no programa HIPERDIA de uma ESF, para verificar o impacto do exercício físico no resultado da EDGA e analisar aspectos sociodemográficos que podem estar relacionados a presença do transtorno depressivo.

A EDGA é um questionário composto por 15 perguntas com respostas objetivas de sim ou não, que de acordo com Brasil (2006), objetiva avaliar a qualidade da saúde mental do idoso, com ênfase na presença de sinais e sintomas do transtorno depressivo, tal escala, utilizada recentemente no estudo de Tavares DMS, et al. (2019), não deve substituir uma entrevista de caráter diagnóstico, realizado por profissionais da área de saúde mental, podendo ser utilizada, apenas com uma ferramenta de avaliação rápida para facilitar a identificação de sinais e sintomas de depressão em idosos. Ainda de acordo com Brasil (2006), a avaliação dos resultados da EDGA consiste na análise dos escores de pontuação, sendo que uma pontuação entre $0 \mathrm{e}$ 5 se considera ausência de depressão, 6 a 10 indica depressão leve e 11 a 15 depressão severa.

A população estudada consistiu em idosos com idade igual ou superior a 60 anos de ambos os gêneros e que estavam cadastrados no programa HIPERDIA ofertado pela ESF . A amostra total foi composta por 59 idosos cadastrados no programa HIPERDIA, pertencentes à área de cobertura da ESF Arenaldo Pinheiro, localizada na zona urbana em um munícipio do PA. A pesquisa foi desenvolvida após a autorização da Secretaria Municipal de Saúde do município e obtenção do parecer consubstanciado favorável, no 3.398.297, do Comitê de Ética em Pesquisa (CEP) envolvendo seres humanos, no dia 18 de junho de 2019.

Como critério de exclusão foi adotado a presença de condições limitantes para realização de exercício físico em seu dia a dia, como: idosos com deficiência física incapacitante, cardiopatias severas, acidente vascular encefálico, acamados, e outras condições que impedem o idoso de praticar exercício físico regularmente. Após se enquadrar nos critérios estabelecidos para participação na pesquisa, o idoso só estava apto a participar da mesma mediante assinatura do Termo de Consentimento Livre e Esclarecido (TCLE). Após aplicação dos instrumentos de coleta de dados e mediante as respostas obtidas no quesito exercício físico, a amostra foi dividida em: Grupo Ativo (GA) e Grupo Sedentário (GS), além disso no quesito referente ao escore na Escala de Depressão Geriátrica Abreviada (EDGA), foram classificados em: Ausência de depressão (G1), depressão leve (G2) e depressão severa (G3).

Os dados coletados foram tabulados, interpretados, processados e analisados por meio da estatística descritiva e inferencial. Para análise dos dados foram utilizados os recursos de computação, por meio do processamento no sistema Microsoft Excel e Statistic Package for Social Sciences (SPSS) versão 24.0, todos em ambiente Windows 7. Neste estudo foi utilizado o teste de verossimilhança do Qui-quadrado de Person e Denver II adotando o nível de significância de $p<0,05$. 


\section{RESULTADOS}

A Tabela 1 mostra que a maioria significativa dos idosos é do sexo feminino (32; $54,2 \%)$, casados (27; $45,8 \%)$, possuem filhos ( $56 ; 94,9 \%)$, moram com parceiro $(25 ; 42,4 \%)$ e filhos $(17 ; 28,8 \%)$, são de cor parda (32; $54,2 \%)$, recebem um salário mínimo $(47 ; 79,7 \%)$ e são alfabetizados $(35 ; 59,3 \%)$.

Tabela 1 - Distribuição dos idosos ativos e sedentários do HIPERDIA, segundo os aspectos sociodemográfico.

\begin{tabular}{|c|c|c|c|}
\hline Caracterização Sociodemográfica & Frequência & Percentual (\%) & P-Valor ${ }^{(1)}$ \\
\hline \multicolumn{4}{|l|}{ Sexo } \\
\hline Feminino & 32 & $54,2 \%$ & \multirow{2}{*}{$0.515 \mathrm{~ns}$} \\
\hline Masculino & 27 & $45,8 \%$ & \\
\hline \multicolumn{4}{|l|}{ Estado Civil } \\
\hline Casado & 27 & $45,8 \%$ & \multirow{4}{*}{$0.000^{*}$} \\
\hline Solteiro & 9 & $15,3 \%$ & \\
\hline União Estável & 5 & $8,5 \%$ & \\
\hline Viúvo & 18 & $30,5 \%$ & \\
\hline \multicolumn{4}{|l|}{ Filhos } \\
\hline Não & 3 & $5,1 \%$ & \multirow{2}{*}{$0.000^{*}$} \\
\hline Sim & 56 & $94,9 \%$ & \\
\hline \multicolumn{4}{|l|}{ Mora Com Quem? } \\
\hline Filhos & 12 & $20,3 \%$ & \multirow{9}{*}{$0.000^{*}$} \\
\hline Irmão & 1 & $1,7 \%$ & \\
\hline Neto & 1 & $1,7 \%$ & \\
\hline Parceiro & 25 & $42,4 \%$ & \\
\hline Parceiro/Bisneto & 1 & $1,7 \%$ & \\
\hline Parceiro/Filho/Neto & 2 & $3,4 \%$ & \\
\hline Parceiro/Filhos & 3 & $5,1 \%$ & \\
\hline Parceiro/Neto/Bisneto & 1 & $1,7 \%$ & \\
\hline Sozinho & 13 & $22,0 \%$ & \\
\hline \multicolumn{4}{|l|}{ Cor } \\
\hline Amarela & 1 & $1,7 \%$ & \multirow{5}{*}{$0.000^{*}$} \\
\hline Branca & 10 & $16,9 \%$ & \\
\hline Não Sabe Declarar & 1 & $1,7 \%$ & \\
\hline Parda & 32 & $54,2 \%$ & \\
\hline Preta & 15 & $25,4 \%$ & \\
\hline \multicolumn{4}{|l|}{ Renda } \\
\hline Abaixo De Um Salário Mínimo & 6 & $10,2 \%$ & \multirow{3}{*}{$0.000^{*}$} \\
\hline Acima De Um Salário Mínimo & 6 & $10,2 \%$ & \\
\hline Um Salário Mínimo & 47 & $79,7 \%$ & \\
\hline \multicolumn{4}{|l|}{ Escolaridade } \\
\hline Alfabetizacão & 35 & $59,3 \%$ & \multirow{3}{*}{$0.000^{*}$} \\
\hline Analfabeto & 12 & $20,3 \%$ & \\
\hline Fundamental & 12 & $20.3 \%$ & \\
\hline
\end{tabular}

Nota: Os resultados são baseados em linhas e colunas não vazias em cada subtabela mais interna. Legenda:

(1) Teste Qui-quadrado (Wilks' G²) de Pearson para independência ( $p$-valor $<0.05)$. *Valores Significativos; NS

- Valores Não Significativos. $\mathrm{H}_{0}$ : As frequências observadas ocorrem na mesma proporção para os diferentes grupos. Ha: As frequências observadas diferem significativamente para os diferentes grupos. Decisão: Como o valor de $p$ computado é menor que o nível de significância alfa $=0,05$, deve-se rejeitar a hipótese nula $\mathrm{H}_{0} \mathrm{e}$ aceitar a hipótese alternativa Há.

Fonte: Melo MG, et al., 2020.

Quanto aos aspectos clínicos e hábitos de vida, a Tabela 2 mostra que a maioria significativa dos idosos possui Hipertensão Arterial Sistêmica (HAS) (31; 52,5\%\%), não são ativos (34; 57,6\%), apenas 25 (42,4\%) praticam atividade física, todos os dias por 60 minutos $(10 ; 16,9 \%)$, além disso, $41(69,5 \%)$ idosos apresentaram escore EDGA entre 1 e 5, indicando ausência de depressão na maioria dos idosos avaliados. 
Tabela 2 - Distribuição dos idosos ativos e sedentários do HIPERDIA, segundo os Aspectos Clínicos e Hábitos de Vida.

\begin{tabular}{|c|c|c|c|}
\hline Aspectos Clínicos e Hábitos de Vida & Frequência & Percentual (\%) & P-Valor ${ }^{(1)}$ \\
\hline \multicolumn{4}{|l|}{ Doença Crônica } \\
\hline Diabetes & 1 & $1,7 \%$ & \multirow{5}{*}{$0.002^{*}$} \\
\hline Diabetes/Outros & 2 & $3,4 \%$ & \\
\hline HAS & 31 & $52,5 \%$ & \\
\hline HAS/Diabetes & 22 & $37,3 \%$ & \\
\hline HAS/Outros & 3 & $5,1 \%$ & \\
\hline \multicolumn{4}{|l|}{ Ativo } \\
\hline Não & 34 & $57,6 \%$ & \multirow{2}{*}{$0.001^{*}$} \\
\hline Sim & 25 & $42,4 \%$ & \\
\hline \multicolumn{4}{|l|}{ Frequência/Min } \\
\hline 3X Semana/60 Min & 8 & $13,6 \%$ & \multirow{5}{*}{$0.000^{*}$} \\
\hline 4X Semana/60 Min & 5 & $8,5 \%$ & \\
\hline 5X Semana/60 Min & 2 & $3,4 \%$ & \\
\hline Não & 34 & $57,6 \%$ & \\
\hline Todos Dias/60 Min & 10 & $16,9 \%$ & \\
\hline \multicolumn{4}{|l|}{ Sedentário } \\
\hline Não & 25 & $42,4 \%$ & \multirow{2}{*}{$0.001^{*}$} \\
\hline Sim & 34 & $57,6 \%$ & \\
\hline \multicolumn{4}{|l|}{ EDGA } \\
\hline Ausência de depressão (escore 1 a 5) & 41 & $69,5 \%$ & \multirow{3}{*}{$0.001^{*}$} \\
\hline Depressão leve (escore 6 a 10) & 16 & $27,1 \%$ & \\
\hline Depressão severa (escore 11 ou mais) & 2 & $3,4 \%$ & \\
\hline
\end{tabular}

Nota: Os resultados são baseados em linhas e colunas não vazias em cada subtabela mais interna. Legenda:

(1) Teste Qui-quadrado (Wilks' G²) de Pearson para independência ( $p$-valor<0.05). *Valores Significativos; NS

- Valores Não Significativos. $\mathbf{H}_{0}$ : As frequências observadas ocorrem na mesma proporção para os diferentes grupos. Ha: As frequências observadas diferem significativamente para os diferentes grupos. Decisão: Como o valor de $p$ computado é menor que o nível de significância alfa $=0,05$, deve-se rejeitar a hipótese nula $\mathrm{H}_{0} \mathrm{e}$ aceitar a hipótese alternativa Há. Fonte: Melo MG, et al., 2020.

Buscando verificar o comportamento do resultado obtido pelos idosos no teste Denver II, diante das características destes idosos, observa-se na Tabela 3 que nos três grupos de resultados Denver, a maioria dos idosos possui idade entre 60 e 69 anos, a saber, G1 (21; 51,2\%), G2 (8; 50\%) e G3 (1;50\%), são do sexo feminino G1 $(21 ; 51,2 \%)$, G2 $(9 ; 56,3 \%)$ e G3 $(2 ; 100 \%)$, quanto ao estado civil dos idosos, observa-se que $21(51,2 \%)$ idosos sem depressão são casados, enquanto que $7(43,8 \%)$ idosos com depressão leve são viúvos, a maioria dos idosos nos três grupos possuem filhos, G1 (40; 97,6\%), G2 (14; 87,5\%) e G3 (2; $100 \%)$, predominou nos três grupos, os idosos que moram com o parceiro, G1 (18; 43,9\%), G2 (6; 37,5\%) e G3 (1; $50 \%)$, cor parda no G1 (22; 53,7\%), G2 $(9 ; 56,3 \%)$ e G3 (1;50\%), renda de 1 salário mínimo nos três grupos no G1 (33; 80,5\%), G2 (13; 81,3\%) e G3 (1; 50\%), escolaridade nível alfabetização nos três grupos G1 (24; $58,5 \%)$, G2 (10; $62,5 \%)$ e G3 (1; $50 \%)$. Quanto às doenças crônicas verifica-se que a maioria dos idosos sem depressão apresentam apenas HAS $(23 ; 56,1 \%)$, enquanto que entre os idosos com depressão leve a maioria possui HAS e Diabetes ( $8 ; 50 \%$ ), os idosos ativos são predominantes no grupo G1 (24; $58,5 \%$ ), enquanto no grupo G2, predominou idosos não ativos (15; 93,8\%), assim como os 2 (100\%) idosos do grupo com depressão severa.

A Tabela 4 mostra quais variáveis possuem maior impacto sobre a resposta obtida na escala Denver II, cujo preditor de referência é a ausência de depressão. Verifica-se na tabela abaixo que a presença de certas características (possuir filhos, morar com o parceiro e ter um estilo de vida ativo) apresentaram valores significativos para o estudo, podendo estar associadas à ausência de depressão na pessoa idosa. De acordo com a tabela 4, aqueles idosos que possuem filhos ou que moram com parceiro, apresentaram respectivamente 2,97 e 2,65 vezes, ou seja, aproximadamente 3 vezes mais chances de ausência de depressão. Da mesma forma, verifica-se que os idosos ativos apresentaram 32,36 vezes mais chance de ter ausência de depressão como resposta no teste de regressão logísitica Ordinal, Denver II. 


\section{Revista Eletrônica Acervo Saúde / Electronic Journal Collection Health ｜ ISSN 2178-2091}

Tabela 3 - Distribuição dos idosos ativos e sedentários do HIPERDIA, segundo a associação entre os aspectos sociodemográficos, clínicos, hábitos de vida e o resultado obtido na Escala de Depressão Geriátrica Abreviada.

\begin{tabular}{|c|c|c|c|c|c|c|}
\hline \multirow{3}{*}{ Caracterização } & \multicolumn{6}{|c|}{ Denver } \\
\hline & \multicolumn{2}{|c|}{ G1 - Ausência de depressão $(n=41)$} & \multicolumn{2}{|c|}{ G2 - Depressão leve $(n=16)$} & \multicolumn{2}{|c|}{ G3 - Depressão severa $(n=2)$} \\
\hline & $\mathbf{n}$ & $\%$ & $\mathbf{n}$ & $\%$ & $\mathbf{n}$ & $\%$ \\
\hline \multicolumn{7}{|l|}{ Faixa Etária } \\
\hline $60-69$ & 21 & $51,2 \%$ & 8 & $50,0 \%$ & 1 & $50,0 \%$ \\
\hline $70-79$ & 19 & $46,3 \%$ & 6 & $37,5 \%$ & 1 & $50,0 \%$ \\
\hline $80-89$ & 1 & $2,4 \%$ & 2 & $12,5 \%$ & 0 & $0,0 \%$ \\
\hline \multicolumn{7}{|l|}{ Sexo } \\
\hline Feminino & 21 & $51,2 \%$ & 9 & $56,3 \%$ & 2 & $100,0 \%$ \\
\hline Masculino & 20 & $48,8 \%$ & 7 & $43,8 \%$ & 0 & $0,0 \%$ \\
\hline \multicolumn{7}{|l|}{ Estado civil } \\
\hline Casado & 21 & $51,2 \%$ & 5 & $31,3 \%$ & 1 & $50,0 \%$ \\
\hline Solteiro & 7 & $17,1 \%$ & 2 & $12,5 \%$ & 0 & $0,0 \%$ \\
\hline União Estável & 3 & $7,3 \%$ & 2 & $12,5 \%$ & 0 & $0,0 \%$ \\
\hline Viúvo & 10 & $24,4 \%$ & 7 & $43,8 \%$ & 1 & $50,0 \%$ \\
\hline \multicolumn{7}{|l|}{ Filhos } \\
\hline Não & 1 & $2,4 \%$ & 2 & $12,5 \%$ & 0 & $0,0 \%$ \\
\hline Sim & 40 & $97,6 \%$ & 14 & $87,5 \%$ & 2 & $100,0 \%$ \\
\hline \multicolumn{7}{|l|}{ Mora com quem? } \\
\hline Filhos & 7 & $17,1 \%$ & 4 & $25,0 \%$ & 1 & $50,0 \%$ \\
\hline Irmão & 1 & $2,4 \%$ & 0 & $0,0 \%$ & 0 & $0,0 \%$ \\
\hline Neto & 1 & $2,4 \%$ & 0 & $0,0 \%$ & 0 & $0,0 \%$ \\
\hline Parceiro & 18 & $43,9 \%$ & 6 & $37,5 \%$ & 1 & $50,0 \%$ \\
\hline Parceiro/Bisneto & 1 & $2,4 \%$ & 0 & $0,0 \%$ & 0 & $0,0 \%$ \\
\hline Parceiro/Filho/Neto & 2 & $4,9 \%$ & 0 & $0,0 \%$ & 0 & $0,0 \%$ \\
\hline Parceiro/Filhos & 3 & $7,3 \%$ & 0 & $0,0 \%$ & 0 & $0,0 \%$ \\
\hline Parceiro/Neto/Bisneto & 0 & $0,0 \%$ & 1 & $6,3 \%$ & 0 & $0,0 \%$ \\
\hline Sozinho & 8 & $19,5 \%$ & 5 & $31,3 \%$ & 0 & $0,0 \%$ \\
\hline \multicolumn{7}{|l|}{ Cor } \\
\hline Amarela & 1 & $2,4 \%$ & 0 & $0,0 \%$ & 0 & $0,0 \%$ \\
\hline Branca & 7 & $17,1 \%$ & 3 & $18,8 \%$ & 0 & $0,0 \%$ \\
\hline Não Sabe Declarar & 1 & $2,4 \%$ & 0 & $0,0 \%$ & 0 & $0,0 \%$ \\
\hline Parda & 22 & $53,7 \%$ & 9 & $56,3 \%$ & 1 & $50,0 \%$ \\
\hline Preta & 10 & $24,4 \%$ & 4 & $25,0 \%$ & 1 & $50,0 \%$ \\
\hline
\end{tabular}

REAS/EJCH | Vol.12(9) | e4214 | DOI: https://doi.org/10.25248/reas.e4214.2020 Página 6 de 13 
Revista Eletrônica Acervo Saúde / Electronic Journal Collection Health ｜ ISSN 2178-2091

\begin{tabular}{|c|c|c|c|c|c|c|}
\hline \multirow{3}{*}{ Caracterização } & \multicolumn{6}{|c|}{ Denver } \\
\hline & \multicolumn{2}{|c|}{ G1 - Ausência de depressão ( $n=41)$} & \multicolumn{2}{|c|}{ G2 - Depressão leve $(n=16)$} & \multicolumn{2}{|c|}{ G3 - Depressão severa $(n=2)$} \\
\hline & $\mathbf{n}$ & $\%$ & $\mathbf{n}$ & $\%$ & $\mathbf{n}$ & $\%$ \\
\hline \multicolumn{7}{|l|}{ Renda } \\
\hline Abaixo De Um Salário Mínimo & 3 & $7,3 \%$ & 2 & $12,5 \%$ & 1 & $50,0 \%$ \\
\hline Acima De Um Salário Mínimo & 5 & $12,2 \%$ & 1 & $6,3 \%$ & 0 & $0,0 \%$ \\
\hline Um Salário Mínimo & 33 & $80,5 \%$ & 13 & $81,3 \%$ & 1 & $50,0 \%$ \\
\hline \multicolumn{7}{|l|}{ Escolaridade } \\
\hline Alfabetização & 24 & $58,5 \%$ & 10 & $62,5 \%$ & 1 & $50,0 \%$ \\
\hline Analfabeto & 7 & $17,1 \%$ & 4 & $25,0 \%$ & 1 & $50,0 \%$ \\
\hline Fundamental & 10 & $24,4 \%$ & 2 & $12,5 \%$ & 0 & $0,0 \%$ \\
\hline \multicolumn{7}{|l|}{ Doença crônica } \\
\hline Diabetes & 1 & $2,4 \%$ & 0 & $0,0 \%$ & 0 & $0,0 \%$ \\
\hline Diabetes/Outros & 2 & $4,9 \%$ & 0 & $0,0 \%$ & 0 & $0,0 \%$ \\
\hline HAS & 23 & $56,1 \%$ & 7 & $43,8 \%$ & 1 & $50,0 \%$ \\
\hline HAS/Diabetes & 13 & $31,7 \%$ & 8 & $50,0 \%$ & 1 & $50,0 \%$ \\
\hline HAS/Outros & 2 & $4,9 \%$ & 1 & $6,3 \%$ & 0 & $0,0 \%$ \\
\hline \multicolumn{7}{|l|}{ Ativo } \\
\hline Não & 17 & $41,5 \%$ & 15 & $93,8 \%$ & 2 & $100,0 \%$ \\
\hline Sim & 24 & $58,5 \%$ & 1 & $6,3 \%$ & 0 & $0,0 \%$ \\
\hline \multicolumn{7}{|l|}{ Frequência/Min } \\
\hline 3x Semana/60 Min & 8 & $19,5 \%$ & 0 & $0,0 \%$ & 0 & $0,0 \%$ \\
\hline 4x Semana/60 Min & 5 & $12,2 \%$ & 0 & $0,0 \%$ & 0 & $0,0 \%$ \\
\hline $5 x$ Semana/60 Min & 2 & $4,9 \%$ & 0 & $0,0 \%$ & 0 & $0,0 \%$ \\
\hline Todos Dias/60 Min & 9 & $22,0 \%$ & 1 & $6,3 \%$ & 0 & $0,0 \%$ \\
\hline Não & 17 & $41,5 \%$ & 15 & $93,8 \%$ & 2 & $100,0 \%$ \\
\hline \multicolumn{7}{|l|}{ Sedentário } \\
\hline Não & 24 & $58,5 \%$ & 1 & $6,3 \%$ & 0 & $0,0 \%$ \\
\hline Sim & 17 & $41,5 \%$ & 15 & $93,8 \%$ & 2 & $100,0 \%$ \\
\hline
\end{tabular}

Fonte: Melo MG, et al., 2020.

REAS/EJCH | Vol.12(9) | e4214 | DOI: https://doi.org/10.25248/reas.e4214.2020 Página 7 de 13 
Tabela 4 - Regressão Logística Ordinal para resultado obtido na Escala de Depressão Geriátrica Abreviada por idosos ativos e sedentários do HIPERDIA, segundo os aspectos sociodemográficos, clínicos, hábitos de vida.

\begin{tabular}{|c|c|c|c|c|c|c|c|}
\hline \multicolumn{5}{|c|}{ Regressão Logística Ordinal } & \multirow{2}{*}{$\begin{array}{l}\text { Razão de } \\
\text { Chances }\end{array}$} & \multicolumn{2}{|c|}{ IC de $95 \%$} \\
\hline Preditor referência = Ausência de depressão & Coef. & $\begin{array}{c}\text { Erro padrão do } \\
\text { Coeficiente }\end{array}$ & $\mathbf{Z}$ & $\mathbf{P}^{(1)}$ & & Inferior & Superior \\
\hline $\begin{array}{l}\text { Const(1) } \\
\text { Const(2) }\end{array}$ & $\begin{array}{l}0,92 \\
3,56\end{array}$ & $\begin{array}{l}0,59 \\
0,90\end{array}$ & $\begin{array}{l}1,56 \\
3,95\end{array}$ & $\begin{array}{l}0,119 \\
0,00{ }^{*}\end{array}$ & & & \\
\hline \multicolumn{8}{|l|}{ Faixa Etária } \\
\hline $\begin{array}{l}70-79 \\
80-89 \\
\end{array}$ & $\begin{array}{c}0,11 \\
-1,58 \\
\end{array}$ & $\begin{array}{l}0,63 \\
1,46 \\
\end{array}$ & $\begin{array}{c}0,17 \\
-1,08\end{array}$ & $\begin{array}{l}0,864 \\
0,279\end{array}$ & $\begin{array}{l}1,11 \\
0,20\end{array}$ & $\begin{array}{l}0,32 \\
0,01\end{array}$ & $\begin{array}{l}3,83 \\
3,61\end{array}$ \\
\hline \multicolumn{8}{|l|}{ Gênero } \\
\hline $\mathrm{M}$ & 0,54 & 0,66 & 0,82 & 0,413 & 1,72 & 0,47 & 6,26 \\
\hline \multicolumn{8}{|l|}{ Estado Civil } \\
\hline Solteiro & 0,05 & 0,94 & 0,05 & 0,959 & 1,05 & 0,17 & 6,66 \\
\hline União Estável & $-0,11$ & 1,29 & $-0,09$ & 0,931 & 0,89 & 0,07 & 11,32 \\
\hline Viúvo & $-0,82$ & 0,68 & $-1,21$ & 0,227 & 0,44 & 0,12 & 1,66 \\
\hline $\begin{array}{l}\text { Const(1) } \\
\text { Const(2) }\end{array}$ & $\begin{array}{l}20,71 \\
23,50\end{array}$ & $\begin{array}{l}26924 \\
26924\end{array}$ & $\begin{array}{l}0 \\
0\end{array}$ & $\begin{array}{l}0,999 \\
0,999\end{array}$ & & & \\
\hline \multicolumn{8}{|l|}{ Filhos } \\
\hline Sim & 1,09 & 1,28 & 0,85 & $0,043^{*}$ & 2,97 & 0,24 & 36,34 \\
\hline \multicolumn{8}{|l|}{ Com Quem Mora? } \\
\hline Irmão & 21,55 & 26924 & 0 & 0,999 & 2,30 & 0 & * \\
\hline Neto & 21,55 & 26924 & 0 & 0,999 & 2,30 & 0 & * \\
\hline Parceiro & 0,97 & 0,87 & 1,12 & $0,024^{*}$ & 2,65 & 0,48 & 14,56 \\
\hline Parceiro/Bisneto & 21,67 & 26924 & 0 & 0,999 & 2,57 & 0 & * \\
\hline Parceiro/Filho/Neto & 21,55 & 19038,2 & 0 & 0,999 & 2,30 & 0 & * \\
\hline Parceiro/Filhos & 21,59 & 15540,5 & 0 & 0,999 & 2,39 & 0 & * \\
\hline Parceiro/Neto/Bisneto & $-1,64$ & 2,22 & $-0,74$ & 0,460 & 0,19 & 0 & 15,13 \\
\hline Sozinho & 0,60 & 0,95 & 0,63 & 0,530 & 1,82 & 0,28 & 11,69 \\
\hline \multicolumn{8}{|l|}{ Cor } \\
\hline Branca & $-21,24$ & 26924 & 0 & 0,999 & 0,00 & 0 & * \\
\hline Não sabe declarar & $-0,97$ & 38076,3 & 0 & 1,000 & 0,38 & 0 & * \\
\hline Parda & $-21,55$ & 26924 & 0 & 0,999 & 0,00 & 0 & * \\
\hline Preta & $-21,67$ & 26924 & 0 & 0,999 & 0,00 & 0 & * \\
\hline
\end{tabular}

REAS/EJCH | Vol.12(9) | e4214 | DOI: https://doi.org/10.25248/reas.e4214.2020 Página 8 de 13 
Revista Eletrônica Acervo Saúde / Electronic Journal Collection Health ｜ ISSN 2178-2091

\section{Regressão Logística Ordinal}

\begin{tabular}{|c|c|c|c|c|c|c|c|}
\hline & \multirow{2}{*}{$\begin{array}{l}\text { Razão de } \\
\text { Chances }\end{array}$} & \\
\hline Preditor referência = Ausência de depressão & Coef. & $\begin{array}{c}\text { Erro padrão do } \\
\text { Coeficiente }\end{array}$ & $\mathbf{Z}$ & $\mathbf{P}^{(1)}$ & & Inferior & Superior \\
\hline \multicolumn{8}{|l|}{ Renda } \\
\hline Acima de um salário mínimo & 0,76 & 1,54 & 0,49 & 0,622 & 2,14 & 0,1 & 44,22 \\
\hline Um salário mínimo & $-0,40$ & 1,13 & $-0,36$ & 0,721 & 0,67 & 0,07 & 6,1 \\
\hline $\begin{array}{l}\text { Const(1) } \\
\text { Const(2) }\end{array}$ & $\begin{array}{l}17,92 \\
20,97\end{array}$ & $\begin{array}{l}26902,2 \\
26902,2\end{array}$ & $\begin{array}{l}0 \\
0\end{array}$ & $\begin{array}{l}0,999 \\
0,999\end{array}$ & & & \\
\hline \multicolumn{8}{|l|}{ Escolaridade } \\
\hline Analfabeto & $-0,33$ & 0,79 & $-0,42$ & 0,677 & 0,72 & 0,15 & 3,43 \\
\hline Fundamental & 0,54 & 1,00 & 0,54 & 0,589 & 1,72 & 0,24 & 12,3 \\
\hline \multicolumn{8}{|l|}{ Doença Crônica } \\
\hline Diabetes/Outros & 3,47 & 32948,3 & 0 & 1,000 & 32,36 & 0 & * \\
\hline HAS & $-17,61$ & 26902,2 & 0 & 0,999 & 0 & 0 & * \\
\hline HAS/Diabetes & $-18,70$ & 26902,2 & 0 & 0,999 & 0 & 0 & * \\
\hline HAS/Outros & $-18,86$ & 26902,2 & 0 & 0,999 & 0 & 0 & * \\
\hline \multicolumn{8}{|l|}{ Ativo } \\
\hline Sim & 3,47685 & 1,13986 & 3,05 & $0,002^{*}$ & 32,36 & 3,47 & 302,15 \\
\hline
\end{tabular}

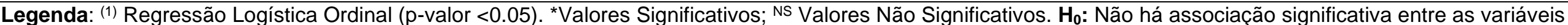

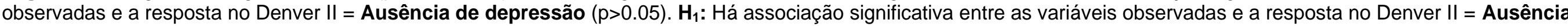
de depressão $(p<0.05)$.

Fonte: Melo MG, et al., 2020. 


\section{DISCUSSÃO}

De acordo com os registros da Estratégia Saúde da Família (ESF) estudada, a quantidade de pacientes cadastrados no programa de Hipertensão e Diabetes (HIPERDIA) é de aproximadamente 300, porém não eram estratificados por idade. Durante a realização da pesquisa, verificou-se que grande parcela dos pacientes tinha idade inferior a 60 anos. De acordo com o Departamento de Informática do Sistema Único do SUS (DATASUS), entre os anos de 2002 e 2013, haviam aproximadamente 1.823 pacientes cadastrados no programa HIPERDIA, na cidade de Conceição do Araguaia-PA, sendo que 974 pessoas apresentavam idade superior a 60 anos (BRASIL, 2013). Um fator que pode justificar o pequeno tamanho amostral ( $n=59)$ é que os pacientes idosos não frequentam com assiduidade as consultas do programa, por motivos variados dentre eles, a compra do próprio medicamento em farmácias particulares ou a ida na ESF por interesse exclusivo na receita médica.

Do total de idosos participantes da pesquisa, a maioria era do gênero feminino (32; $54,2 \%$ ) que pode ser explicado por alguns fatores, entre eles, a população idosa ser composta em sua maioria pelo gênero feminino (56\%) de acordo o IBGE (2018), bem como a maior procura desse gênero aos serviços de saúde e maior preocupação com a saúde em comparação com o sexo masculino, o que gera maior adesão aos programas de saúde (ALMEIDA E, et al., 2018; MAXIMINIANO-BARRETO MA, et al., 2019; TAVARES DMS, et al., 2019; AGUIAR BM, et al., 2019). Outra possível explicação para o percentual feminino ser maior, é a predominância nos índices de mortalidade masculina, o que por consequência gera uma feminização da velhice (DONATTI A, et al., 2019).

Quanto ao estado civil, a maioria ser casado $(27 ; 45,8 \%)$ também está de acordo com os dados do IBGE (2018), que demostram que $56,3 \%$ da população brasileira com mais de 15 anos é casada; e no estado do Pará, essa média é de aproximadamente $56,5 \%$, sendo composta por pessoas com casamento civil/religioso $(26,7 \%)$ e união consensual $(29,8 \%)$. Esse resultado também foi encontrado em outros estudos (CAMBOIM FEF, et al., 2017; LEÃO RCH, et al., 2017; SILVA AR, et al., 2017; CABRAL JF, et al., 2019). Dos 59 idosos, 56 idosos $(94,9 \%)$ possuem filhos, o que pode ser entendido pelo fato de que a taxa de fecundidade no país em décadas passadas apresentava valores maiores, como por exemplo, no ano de 1940 a taxa de fecundidade era de aproximadamente 6,16, e diminuiu para 1,90 em 2010 (IBGE, 2010). Essa alta prevalência de idosos com filhos também foi encontrado em outros estudos (GATO JM, et al., 2018; DONATTI A, et al., 2019).

Quanto à cor da pele, a maioria $(32 ; 54,2 \%)$ dos idosos se autodeclaram pardos, o que pode ser explicado pelo processo de miscigenação e a diversidade racial presente no Brasil. De acordo com o IBGE (2010) aproximadamente 80 milhões de brasileiros são pardos. A Pesquisa Nacional por Amostra de Domicílio (PNAD) realizada pelo Instituto Brasileiro de Geografia e Estatística (IBGE, 2016) demonstrou que na região norte cerca de $72,3 \%$ das pessoas se autodeclaram pardas. A mesma pesquisa demonstra que houve redução de $1,8 \%$ no quantitativo da cor autodeclarada branca, enquanto que a cor autodeclarada preta cresceu $14,9 \%$ e a parda cresceu cerca de $6,6 \%$.

Em relação à condição econômica, pela faixa etária (maior de 60 anos), era esperado que a maioria fosse aposentada, e, de fato, 47 idosos $(79,7 \%)$ recebiam o benefício da aposentadoria pelo Instituto Nacional do Seguro Social (INSS), que concede a aposentadoria por idade rural àquele cidadão que comprove no mínimo 180 meses de contribuições, com a idade mínima de 55 anos para mulheres e 60 anos para homens; e por idade urbana, sendo concedido o benefício para os que tiveram até 180 contribuições, exigindo a idade mínima de 60 anos para as mulheres e 65 para os homens. De acordo com os dados da PNAD Contínua (IBGE, 2016), cerca de 75,6\% dos idosos brasileiros eram aposentados ou pensionistas em 2015.

No quesito escolaridade, a maioria da amostra pesquisada apresentou o percentual de $59,3 \%(n=35)$ de idosos que cursaram somente as primeiras séries da alfabetização. Tal condição pode ser compreendida pelo fato de que nas décadas passadas a inserção em atividades trabalhistas ocorria de forma muito precoce, muitas vezes ainda na infância, com $24,7 \%$ dos idosos ocupados que começaram a trabalhar com menos de 
9 anos de idade e $43 \%$ com idade entre 10 a 14 anos, de acordo com os dados da PNAD Contínua (IBGE, 2016).

A doença mais comum entre os idosos participantes, foi a HAS representando um total de $52,5 \%$. Essa condição clínica está associada principalmente ao envelhecimento e a maus hábitos de vida como: tabagismo, obesidade, etilismo, consumo excessivo de sal e sedentarismo (SANTANA BS, et al., 2019). Uma pesquisa realizada pela Vigilância de Fatores de Risco e Proteção Para Doenças Crônicas por Inquérito Telefônico (VIGITEL) realizado por Brasil (2018), demonstrou que a HAS cresceu de 22,6\% em 2006 para 24,3\% em 2017 entre a população brasileira, porém entre os idosos observa-se que a incidência era maior entre os que apresentavam 65 anos ou mais, cerca de $60,9 \%$.

No quesito atividade física, os idosos apresentaram em sua maioria, um estilo de vida sedentário, que correspondeu a $57,6 \%$ dos participantes, esse achado pode estar associado ao processo de exclusão social, muitas vezes inerente à pessoa idosa, fragilidade, medo de queda, ausência de orientação e/ou estímulos para prática de exercícios físicos de forma regular e condições incapacitantes e debilitantes associadas a velhice, esses fatores na maioria das vezes contribuem para um estilo de vida sedentário (CAMBOIM FEF, et al., 2017).

Um importante dado que conseguiu-se demonstrar com essa pesquisa, foi que $21(51,2 \%)$ idosos sem depressão são casados, enquanto que $7(43,8 \%)$ idosos com depressão leve são viúvos, o que permitiu verificar que os idosos que moram com seus parceiros, têm 2,65 $=3$ vezes mais chances de ter ausência de sinais e sintomas depressivos conforme resposta no Denver II. Tais achados corroboram com o estudo de Gato JM, et al. (2018), que em sua pesquisa constata a associação significativa entre estado civil e depressão, pois os idosos casados, foram classificados sem depressão, ao passo que os divorciados/separados/desquitados e viúvos apresentam depressão moderada.

Além disso, Gullich I, et al. (2016), demonstraram que os idosos casados dispõem de maior interação social e familiar, e a presença dessas relações auxiliam o idoso a tornar-se mais ativo e consequentemente apresente menores chances de desenvolver sinais e sintomas do estado depressivo. Outros estudos constatam que estar casado confere proteção ao desenvolvimento de sinais e sintomas da depressão, em relação aos outros estados civis, pois o fato de estar casado contribui significativamente para um melhor quadro de saúde no idoso, melhorando o enfrentamento de situações e permitindo o apoio mútuo (MINGHELLI B, et al., 2013; SILVA AR, et al., 2017; GATO JM, et al., 2018; MAXIMINIANO-BARRETO MA, et al., 2019).

No que diz respeito à presença de uma ou mais doenças crônicas, foi evidenciado que os idosos pertencentes ao grupo G1 (ausência de depressão), apresentaram em sua maioria (23;56,1\%) apenas a HAS como doença crônica, e no grupo G2 (depressão leve) grande parte $(8 ; 50 \%)$ possuíam 2 doenças crônicas, HAS e Diabetes, o que de certa forma, por mais que a amostra seja pequena, destaca que a presença de duas doenças crônicas pode estar relacionada ao surgimento de sinais e sintomas depressivos. Amaral TLM, et al. (2018) destacam que idosos com multimorbidade apresentaram prevalência de depressão 2 vezes maior do que entre os idosos sem multimorbidade, demonstrando o impacto que tal condição clínica gera na saúde afetiva e humor, que estão associadas à depressão. Mendes-Chiloff CL, et al. (2018) evidenciaram que a prevalência de sinais e sintomas depressivos aumenta conforme a quantidade de doenças autorreferidas, sendo que os idosos que relataram a presença de pelo menos uma doença crônica, tem aproximadamente $1,44 \%$ mais chances de ter sinais e sintomas da depressão, os que relataram possuir duas ou mais doenças crônicas aumentam as chances de apresentar sinais e sintomas depressivos em $2,25 \%$, em comparação aos idosos que não apresentam comorbidades.

Quanto à condição social de ter filhos, a maioria dos idosos que compõem os três grupos da pesquisa possuem filhos, G1 97,6\% (40), G2 87,5\% (14) e G3 100\% (2), podendo estar relacionado ao índice de filhos nas famílias brasileiras, que nas décadas passadas eram altos, essa variável também foi apontada no estudo de Campos ACV, et al. (2016), onde $82,7 \%$ dos idosos tinham dois ou mais filhos. 
O ambiente composto por cônjuges, filhos e familiares proporcionam para o idoso a conservação das redes sociais, preservação dos laços afetivos e estímulos para a independência do idoso. Matos FS, et al. (2018) e Mocellin D, et al. (2019) referem que o cuidado direcionado aos pais no processo de envelhecimento é uma forma de compensação pelos cuidados que receberam anteriormente.

Quanto à associação da prática regular de exercício físico com a manifestação de sinais e sintomas depressivos, os idosos ativos têm 32,36 = 32 vezes mais chance de ter ausência de depressão como resposta no Denver II.

Silva MR, et al. (2018) e Teixeira CM, et al. (2016) verificaram essa relação, onde os idosos sedentários apresentaram maior prevalência de sintomas depressivos, situação que pode ser explicada pelo fato de que a prática de exercício físico gera maior autonomia e melhora na qualidade de vida do idoso, aumenta a capacidade funcional, ameniza os efeitos negativos oriundos do processo de envelhecimento, promove relações sociais, estimula funções cognitivas e previne o surgimento de determinadas perturbações mentais como a depressão.

Groppo HS, et al. (2012) demostraram melhora significativa nos sinais e sintomas depressivos no grupo de idosos com algum nível de depressão que participaram de um programa de exercício físico e concluíram que o paciente depressivo teve sua autoestima elevada; e que o processo de exercitar-se serve como distração para pensamentos negativos e causa efeitos fisiológicos, aumentando a liberação de endorfinas e monoaminas que irão agir diminuindo a ansiedade, tensão e estresse, contribuindo dessa forma para amenização de sinais e sintomas depressivos.

Antunes HKM, et al. (2014) evidenciaram que os idosos submetidos a prática regular de exercícios físicos aeróbios de intensidade moderada, como a caminhada e exercício físico realizado em cicloergômetro, obtiveram os melhores resultados na diminuição do escore de sinais e sintomas depressivos; destacaram ainda que os benefícios do exercício físico se estendem também como uma medida não farmacológica, de suma importância para ao tratamento da depressão, promovendo mudanças no estilo de vida e eliminando sintomas desagradáveis da depressão.

\section{CONCLUSÃO}

De acordo com os resultados, a maioria dos idosos que praticam exercício físico de forma regular, apresentam baixo escore na Escala de Depressão Geripatrica Abrevida (EDGA), indicando que o exercício físico desempenha um importante papel na saúde do idoso, sendo um fator de proteção para o transtorno depressivo. Enquanto parte dos idosos sedentários, apresentam escores elevados na EDGA, indicando depressão leve ou severa. Portanto, a prática de exercício físico proporciona bem-estar físico, emocional e social, mas é válido destacar que, a ausência de depressão pode estar associada também a variáveis sociodemográficas, como morar com o parceiro e ter filhos. Apesar de tais achados, faz-se necessário a realização de estudos com maior número amostral e método com mais acurácia quanto ao estilo de vida, ativo ou sedentário, do participante. Considerando o contexto demográfico brasileiro, onde o número de idosos cresce a cada dia, são necessários os estudos que buscam estratégias de saúde que centrem o cuidado na pessoa idosa, que levem em consideração seu contexto biopsicossocial, ofertando um cuidado eficaz, com foco na prevenção de agravos e promoção de saúde e bem-estar.

\section{REFERÊNCIAS}

1. AGUIAR BM, et al. Avaliação da incapacidade funcional e fatores associados em idosos. Revista Braileira de Geriatria e Gerontolongia, 2019; 22(2): e180163.

2. ALMEIDA E, et al. Saúde mental em idosos brasileiros: efeito de diferentes programas de atividade física. Revista de Psicologia, Saúde e Doenças, 2018; 19(2): 390-404.

3. AMARAL TLM, et al. Multimorbidade, depressão e qualidade de vida em idosos atendidos pela Estratégia de Saúde da Família em Senador Guiomard, Acre, Brasil. Revista Ciência e Saúde coletiva, 2018; 23(9): 3077-3084. 
4. ANTUNES HKM, et al. O baixo consumo de oxigênio tem reflexos nos escores de depressão em idosos. Revista Brasileira de Geriatria e Gerontologia, 2014; 17(3):505-515.

5. ANTUNES PC, et al. Revisão sistemática sobre práticas corporais na perspectiva das práticas integrativas e complementares em saúde. Motrivivência, 2018; 30(55):227-247.

6. BRASIL. Ministério da Saúde. Secretaria de Atenção à Saúde. Departamento de Atenção Básica. Cadernos de Atenção Básica, n. 19. Envelhecimento e saúde da pessoa idosa. Brasília, 2006; 142p.

7. BRASIL. Ministério da Saúde. Sistema de Cadastramento e Acompanhamento de Hipertensos e Diabéticos, Conceição do Araguaia - Pará, 2013. TABNET. Disponível em: http://tabnet.datasus.gov.br/cgi/tabcgi.exe?hiperdia/cnv/hdpa.def.

8. BRASIL. Ministério da Saúde. Secretaria de Vigilância em Saúde. Departamento de Vigilância de Doenças e Agravos não Transmissíveis e Promoção da Saúde. Vigitel Brasil 2017: vigilância de fatores de risco e proteção para doenças crônicas por inquérito telefônico: estimativas sobre frequência e distribuição sociodemográfica de fatores de risco e proteção para doenças crônicas nas capitais dos 26 estados brasileiros e no Distrito Federal em 2017 / Ministério da Saúde, Secretaria de Vigilância em Saúde, Departamento de Vigilância de Doenças e Agravos não Transmissíveis e Promoção da Saúde. Brasília: Ministério da Saúde, 2018; 130p.

9. CABRAL JF, et al. Vulnerabilidade e fatores associados em idosos atendidos pela Estratégia Saúde da Família. Revista Ciência e Saúde Coletiva, 2019; 24(9):3227-3236.

10. CAMBOIM FEF, et al. Benefícios da atividade física na terceira idade para a qualidade de vida. Revista de Enfermagem UFPE on line, $2017 ; 11(6): 2415-2422$.

11. CAMPOS ACV, et al. Perfil do envelhecimento saudável de idosos brasileiros octogenários. Revista Latino-Amricana de Enfermagem, 2016; 24: e2724.

12. DONATTI A, et al. Relação entre a intensidade de dor lombar crônica e limitações geradas com os sintomas depressivos. BrJP, 2019; 2(3): 247-254.

13. GATO JM, et al. Saúde mental e qualidade de vida de pessoas idosas. Avances en Enfermaria, 2018; 36(3):302-310.

14. GIL IMA, et al. Eficácia da reminiscência na cognição, sintomas depressivos e qualidade de vida em idosos na comunidade: revisão sistemática. Revista de Enfermagem Referência, 2018; 4(16):155-160.

15. GROPPO HS, et al. Efeitos de um programa de atividade física sobre os sintomas depressivos e a qualidade de vida de idosos com demência de Alzheimer. Revista Brasileira de Educação Física e Esporte, 2012; 26(4):543-551.

16. GULLICH I, et al. Depressão entre idosos: um estudo de base populacional no Sul do Brasil. Revista Brasileira de Epidemiologia, 2016; 19(4):691-701.

17. INSTITUTO BRASILEIRO DE GEOGRAFIA E ESTATÍSTICA (IBGE). Senso demográfico 2010, projeção 2010/2060. Brasília, 2010. Disponível em: <https://www.ibge.gov.br/estatisticas-novoportal/sociais/populacao/9109-projecao-dapopulacao.html?=\&t=downloads $>$.

18. INSTITUTO BRASILEIRO DE GEOGRAFIA E ESTATÍSTICA (IBGE). Diretoria de Pesquisas, Coordenação de Trabalho e Rendimento, Pesquisa Nacional por Amostra de Domicílios Contínua 2012/2016. 2016. Disponível em: https://biblioteca.ibge.gov.br/visualizacao/livros/liv101377 informativo.pdf.

19. INSTITUTO BRASILEIRO DE GEOGRAFIA E ESTATÍSTICA (IBGE). Agência IBGE notícias. 2018. Disponível em: $<$ https://agenciadenoticias.ibge.gov.br/agencianoticias/ 2012-agencia-de-noticias/noticias/20980-numero-de-idosos-cresce18-em-5-anos-e-ultrapassa-30-milhoes-em-2017>

20. LEÃO RCH, et al. Análise de Classes Latentes: um novo olhar sobre o fenômeno depressão em homens idosos no nordeste do Brasil. Revista Brasileira de Geriatria e Gerontologia, 2017; 20(6):814-825.

21. MATOS FS, et al. Redução da capacidade funcional de idosos residentes em comunidade: estudo longitudinal. Ciência e Saúde Coletiva, 2018; 23(10):3393-3401.

22. MAXIMIANO-BARRETO MA, et al. Ansiedade e depressão e a relação com a desigualdade social entre idosos. Psicologia, Saúde e Doenças, 2019; 20(1):209-219.

23. MENDES-CHILOFF CL, et al. Sintomas depressivos em idosos do município de São Paulo, Brasil: prevalência e fatores associados (Estudo SABE). Revista Brasileira de Epidemiologia, 2018; 21(Suppl 2): e180014.

24. MINGHELLI B, et al. Comparação dos níveis de ansiedade e depressão entre idosos ativos e sedentários. Revista de Psiquiatria Clínica, 2013; 40(2):71-76.

25. MOCELLIN D, et al. Responsabilidade filial: quais as atitudes dos filhos sobre a institucionalização dos pais idosos? Revista Gaúcha de Enfermagem, 2019; 40:e20180377.

26. NASCIMENTO PPP, BATISTONI SST. Depressão e fragilidade na velhice: uma revisão narrativa das publicações de 20082018. Interface (Botucatu), 2019; 23:e180609.

27. NERY BLS, et al. Vulnerabilidades, depressão e religiosidade em idosos internados em uma unidade de emergência. Revista Gaúcha de Enfermagem, 2018; 39:e2017-0184.

28. RODRIGUES RAP, et al. Síndrome da fragilidade entre idosos e fatores associados: comparação de dois municípios. Revista Latino-Americana de Enfermagem, 2018; 26:e3100.

29. SANTANA BS, et al. Hipertensão arterial em idosos acompanhados na atenção primária: perfil e fatores associados. Revista Escola Anna Nery, 2019; 23(2):e20180322.

30. SILVA AR, et al. Doenças crônicas não transmissíveis e fatores sociodemográficos associados a sintomas de depressão em idosos. Jornal Brasileiro de Psiquiatria, 2017; 66(1):45-51.

31. SILVA MR, et al. Sintomas depressivos em idosos e sua relação com dor crônica, doenças crônicas, qualidade do sono e nível de atividade física. BrJP, 2018; 1(4):293-298.

32. SILVA VRR, et al. Benefícios do exercício físico sobre as alterações fisiológicas, aspectos sociais, cognitivos e emocionais no envelhecimento. Revista Centro de pesquisas avançadas em qualidade de vida, 2015; 7(3):1-12.

33. TAVARES DMS, et al. Prevalência de morbidades autorreferidas e fatores associados entre idosos comunitários de Uberaba, Minas Gerais, Brasil. Revista Ciência e Saúde Coletiva, 2019; 24(9): 3305-3313.

34. TEIXEIRA CM, et al. Atividade física, autoestima e depressão em idosos. Cuadernos de Psicología del Deporte, 2016; 16(3): $55-66$. 